\title{
Dynamics and Ulam Stability for Fractional $q$-Difference Inclusions via Picard Operators Theory
}

\author{
Saïd Abbas, Mouffak Benchohra and Erdal Karapınar*
}

\begin{abstract}
In this manuscript, by using weakly Picard operators we investigate the Ulam type stability of fractional $q$-difference An illustrative example is given in the last section.
\end{abstract}

\section{Introduction}

Not only fractional differential inclusions (FDIs) but also fractional differential equations (FDEs) have applications in mathematics, and other applied sciences, see e.g. $[18,6,7,35,38,40,21,22,37,9,17,4,5]$. Fractional $q$ difference equations received much attention from many authors; see e.g. [12]. Other interesting results about this subject can be found in [24].

Functional differential inclusions and coupled systems of differential inclusions are a generalization of the concept of ordinary differential equation of the form $\frac{d}{d t} x(t) \in F(t, x(t))$, where $F$ is a multivalued map containing one element (single-valued map). Differential inclusions arise in many situations as differential variational inequalities, projected dynamical systems, linear and nonlinear complementarity dynamical systems, discontinuous ordinary differential equations, and fuzzy set arithmetic; see e.g.[14, 19, 36].

Key Words: Fractional $q$-difference inclusion; multivalued weakly Picard operator; UlamRassias stability; fixed point inclusion.

2010 Mathematics Subject Classification: Primary 26A33,34A60,Secondary 46T99,47H10

Received: 31.12.2020

Accepted: 19.02.2021

*Corresponding Author 
Ulam stability for functional differential equations and inclusions has been widely considered; see e.g. $[26,27]$. Picard operators [28, 29] seemed to be a powerful method in the processing of Ulam stability theory $[10,27,16]$, and ordinary differential inclusions and equations; see e.g.[1, 29, 30, 31].

In this paper we first discuss the stability of the fractional $q$-difference inclusion below in the sense of Ulam-Rassias

$$
\left({ }^{c} \mathfrak{D}_{q}^{\alpha} \mathfrak{h}\right)(\mathfrak{t}) \in F(\mathfrak{t}, \mathfrak{h}(\mathfrak{t})) ; \mathfrak{t} \in \mathfrak{J}:=[0, T],
$$

along the initial condition

$$
\mathfrak{h}(0)=\mathfrak{h}_{0} \in \mathbb{R}
$$

with $T>0, \alpha \in(0,1], q \in(0,1)$, and $F: \mathfrak{J} \times \mathbb{R} \rightarrow \mathcal{N}(\mathbb{R})$ is a given multi-valued map, $\mathcal{N}(\mathbb{R})$ is the family of all nonempty subsets of $\mathbb{R}$, and ${ }^{c} \mathfrak{D}_{q}^{\alpha}$ is the Caputo fractional $q$-difference derivative of order $\alpha$.

After getting a solution of (1.1), we shall investigate the coupled fractional $q$-difference inclusions

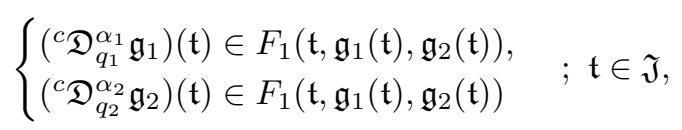

with the initial conditions

$$
\left\{\begin{array}{l}
\mathfrak{g}_{1}(0)=\mathfrak{i}_{1} \\
\mathfrak{g}_{2}(0)=\mathfrak{i}_{2},
\end{array}\right.
$$

where $T>0, q_{i} \in(0,1), \alpha_{i} \in(0,1], \mathfrak{i}_{i} \in \mathbb{R}, F_{i}: \mathfrak{J} \times \mathbb{R} \rightarrow \mathcal{N}(\mathbb{R}) ; i=1,2$.

This paper initiates the application of Picard operators for the study of Ulam stability for problems (1.1)-(1.2) and (1.3)-(1.4).

\section{Preliminaries}

We deal with the following collection

$$
C(\mathfrak{J}):=\{\mathfrak{g}: \mathfrak{J} \rightarrow \mathbb{R} \mid \mathfrak{g} \text { is continuous }\} .
$$

Then, $C(\mathfrak{J})$ forms a Banach space by regarding the supremum (uniform) norm $\|\mathfrak{g}\|_{C}:=\sup _{\tau \in \mathfrak{J}}|\mathfrak{g}(\tau)|$.

$$
L^{1}(\mathfrak{J}):=\{\mathfrak{g}: \mathfrak{J} \rightarrow \mathbb{R} \mid \mathfrak{g} \text { is measurable and Lebesgue integrable function. }\}
$$

Then, $L^{1}(\mathfrak{J})$ forms a Banach space by regarding $\|\mathfrak{g}\|_{L^{1}}=\|\mathfrak{g}\|_{1}=\int_{\mathfrak{J}}|\mathfrak{g}(\tau)| d \tau$. 
Over a metric space $(\mathfrak{M}, \delta)$, the symbol $\mathfrak{P}(E)$ denotes the family of all nonempty subsets of $E \subset \mathfrak{M}$. Then, we set

$$
\mathfrak{P}_{\pi}(E)=\{F \in \mathfrak{P}(E): F \text { fulfills the property } \pi\} \text {, }
$$

where, $\pi$ can be, for instance, bounded, closed, compact, convex (in short, $b d, c l, c p, c v)$. For clarification, consider, for example $\mathfrak{P}_{b d, c l}(E)=\{F \in \mathfrak{P}(E): F$ is bounded and closed $\}$.

A multivalued function $G: \mathfrak{J} \rightarrow \mathfrak{P}_{c l}(E)$ is called measurable whenever the mapping

$$
\tau \rightarrow \operatorname{dist}(\mathfrak{u}, G(\mathfrak{t}))=\inf \{\|\mathfrak{u}-\nu\|: \nu \in G(\tau)\}
$$

is measurable for each $\mathfrak{u} \in E$.

A mapping $H_{d}: \mathfrak{P}(E) \times \mathfrak{P}(E) \rightarrow[0, \infty) \cup\{\infty\}$ described by

$$
H_{d}(A, B)=\max \left\{\sup _{a \in A} d(a, B), \sup _{b \in B} d(A, b)\right\},
$$

is called Hausdorff metric, where $d(a, B)=\inf _{b \in B} d(a, b), d(A, b)=\inf _{a \in A} d(a, b)$ and $A, B \subset E$. Then, the coupled $\left(\mathfrak{P}_{b d, c l}(E), H_{d}\right)$ is named as Hausdorff metric space.

Definition 2.1. [14] The set

$$
S_{G}=\left\{\mathfrak{g} \in L^{1}(\mathfrak{J}): \mathfrak{g}(\tau) \in G(\tau), \text { a.e. } \tau \in \mathfrak{J}\right\},
$$

is the selection set of $G$. Moreover, the set selector $S_{F \circ \mathfrak{g}}$, for each $\mathfrak{g} \in C(\mathfrak{J})$ from $F \circ \mathfrak{g}$ is formulated by $S_{F \circ \mathfrak{g}}:=\left\{\mathfrak{u} \in L^{1}(\mathfrak{J}): \mathfrak{u}(\tau) \in F(\tau, \mathfrak{g}(\tau))\right.$, a.e. $\left.\tau \in \mathfrak{J}\right\}$

A selfmapping $\mathcal{O}$ on a metric space $(\mathfrak{M}, \delta)$ is called

(P.o.) Picard operator (P.o.) if $\mathcal{F}_{\mathcal{i} \chi_{\mathcal{O}}}=\left\{\mathfrak{z}^{*}\right\}$ for $\mathfrak{z}^{*} \in \mathfrak{M}$ and $\left(\mathcal{O}^{n}\left(\mathfrak{z}_{0}\right)\right)_{n \in \mathbb{N}} \rightarrow \mathfrak{z}^{*}$ for any $\mathfrak{z}_{0} \in \mathfrak{M}$.

(w.P.o.) weakly Picard operator (w.P.o) if $\left(\mathcal{O}^{n}(\mathfrak{z})\right)_{n \in \mathbb{N}} \rightarrow \mathfrak{z}^{*} \in \mathfrak{M}$, in a way that $\mathfrak{z}^{*} \in \mathcal{F}_{i} x_{\mathcal{O}}$, (limit may depend on $\mathfrak{z}$ ).

(k.w.P.o.) k-weakly Picard operator (c.w.P.o) if it is (w.P.o) and $d\left(\mathfrak{z}, \mathcal{O}^{\infty}(\mathfrak{z})\right) \leq k d(\mathfrak{z}, \mathcal{O}(\mathfrak{z})) ; \mathfrak{z} \in X$.

where $\mathcal{F}_{\mathfrak{i}} \mathfrak{\mathcal { O }}_{\mathcal{O}}=\left\{\mathfrak{z}: \mathfrak{z}=\mathcal{O}_{\mathfrak{z}}\right\}$. Further, for a (w.P.o) $\mathcal{O}$, we set $\mathcal{O}^{\infty}=\mathcal{O}^{\infty}(\mathfrak{z})=$ $\lim _{n \rightarrow \infty} \mathcal{O}^{n}(\mathfrak{z})$. Notice that $\mathcal{O}^{\infty}(\mathfrak{M})=\mathcal{F}_{\mathcal{i}} \boldsymbol{x}_{\mathcal{O}}$.

A multivalued mapping $\mathcal{Q}: \mathfrak{M} \rightarrow \mathfrak{P}(\mathfrak{M})$ on $(\mathfrak{M}, \delta)$ is called weakly Picard operator (m.w.P.o.) $[23,32]$, if for each $\mathfrak{g} \in \mathfrak{M}$ and $\mathfrak{y} \in \mathcal{Q}(x)$, there is $\left(\mathfrak{g}_{n}\right)_{n \in \mathbb{N}}$ where 
(i) $\mathfrak{g}_{0}=\mathfrak{g}, \mathfrak{g}_{1}=\mathfrak{y}$;

(ii) $\mathfrak{g}_{n+1} \in \mathcal{Q}\left(\mathfrak{g}_{n}\right), n \in \mathbb{N}$;

(iii) $\left(\mathfrak{g}_{n}\right)_{n \in \mathbb{N}} \rightarrow \mathfrak{g}^{*}$ so that $\mathfrak{g}^{*} \in \mathcal{F} \mathfrak{x}_{\mathbf{Q}}$.

Set $\mathfrak{A}:=\left\{\varphi:[0, \infty) \rightarrow[0, \infty) \mid \varphi\right.$ increasing, and $\lim _{n \rightarrow \infty} \varphi^{n}(t) \rightarrow 0$ for every $t \in[0, \infty\}$, where $\varphi^{n}$ is the $n$-th iterate of $\varphi$. Here $\varphi$ is called comparison function [34]. If $\varphi \in \mathfrak{A}$ then $\varphi$ is continuous at 0 and $\varphi(t)<t$ for all $t>0$. Furthermore, we set

$$
\mathfrak{S}:=\left\{\varphi:[0, \infty) \rightarrow[0, \infty) \mid \varphi \text { strictly increasing\& } \sum_{n=1}^{\infty} \varphi^{n}(t)<\infty \text { for all } t \in[0, \infty)\right\},
$$

Here, $\varphi \in \mathfrak{S}$ is called strictly comparison function and $\mathfrak{S} \subset \mathfrak{A}$.

Definition 2.2. For $\varphi \in \mathfrak{A}$, operator $\mathcal{Q}: \mathfrak{M} \rightarrow \mathfrak{P}_{c l}(\mathfrak{M})$ is called $\varphi$-multivalued weakly Picard (briefly $\varphi-m . w . P$. operator) if it is a m.w.P. and there is a selection $\mathcal{O}^{\infty}: \Lambda_{\mathcal{Q}} \rightarrow \mathcal{F}_{\text {ix }}$ of $\mathcal{Q}^{\infty}$ so that

$$
d\left(\theta, \mathcal{O}^{\infty}(\theta, \nu)\right) \leq \varphi(d(\theta, \nu)) ; \text { for all }(\theta, \nu) \in \Lambda_{\mathcal{Q}}
$$

In particular, if $\varphi(\mathfrak{z})=k_{\mathfrak{z}}$, for all $\mathfrak{z} \in \mathbb{R}_{+}$, for some $k>0$ then $\mathcal{Q}$ is named as $k$-multivalued weakly Picard operator (k-m.w.P.o.).

Definition 2.3. An operator $\mathcal{Q}: \mathfrak{M} \rightarrow \mathfrak{P}_{c l}(\mathfrak{M})$ is named

a) multivalued $k$-Lipschitz if there is $k \geq 0$ with

$$
H_{\delta}(\mathcal{Q}(\mathfrak{q}), \mathcal{Q}(\nu)) \leq \gamma \delta(\mathfrak{q}, v) ; \text { for each } \mathfrak{q}, \nu \in \mathfrak{M}
$$

b) a multivalued $k$-contraction if (2.1) holds for $k \in[0,1)$,

c) a multivalued $\varphi$-contraction if there is a $\varphi \in \mathfrak{S}$ with

$$
H_{\delta}(\mathcal{Q}(\mathfrak{q}), \mathcal{Q}(\nu)) \leq \varphi(\delta(\mathfrak{q}, \nu)) ; \text { for each } \mathfrak{q}, \nu \in \mathfrak{M}
$$

Definition 2.4. [1]. The inclusion $\mathfrak{g} \in \mathcal{Q}(\mathfrak{g})$ is named generalized Ulam type (g.U.t) stable if there is $\varphi \in \mathfrak{S}$ such that for each $\varepsilon>0$ and solution $\mathfrak{g} \in C(\mathfrak{J})$ of

$$
H_{\delta}(\mathfrak{g}(\tau),(\mathcal{Q g})(\tau)) \leq \varepsilon ; \tau \in \mathfrak{J}
$$

there is a solution $\mathfrak{u} \in C(\mathfrak{J})$ of $\mathfrak{g} \in \mathcal{Q}(\mathfrak{g})$ (inclusion) so that

$$
|\mathfrak{g}(\tau)-\mathfrak{u}(\tau)| \leq \theta_{\mathcal{Q}}(\varepsilon) ; \tau \in \mathfrak{J} .
$$

In case of $\varphi(t)=k t ; k>0$, it is called Ulam type stable. 
Definition 2.5. [1, 2, 3]. The fixed point inclusion $\mathfrak{g} \in \mathcal{Q}(\mathfrak{g})$ is named generalized Ulam-Rassias type stable with respect to $\phi$ if there is a real number $c_{N, \phi}>0$ such that for each solution $\mathfrak{g} \in C_{\gamma}$ of

$$
H_{d}(\mathfrak{g}(\tau),(\mathcal{Q g})(\tau)) \leq \phi(\tau) ; t \in I,
$$

there is a solution $\mathfrak{u} \in C(\mathfrak{J})$ of $\mathfrak{g} \in \mathcal{Q}(\mathfrak{g})$ (inclusion) such that

$$
|\mathfrak{g}(\tau)-\mathfrak{u}(\tau)| \leq c_{N, \phi} \phi(\tau) ; t \in I .
$$

In case of $\phi(t)=k t ; k>0$, it is called Ulam-Rassias type stable.

Lemma 2.6. [39] Let $\mathcal{Q}$ be a multivalued mapping from a complete metric space $(\mathfrak{M}, \delta)$ to $\mathfrak{P}_{c l}(\mathfrak{M})$, and $\lambda \in \mathfrak{S}$. If $\mathcal{Q}$ is a $\lambda$-contraction, then $\mathcal{F}_{\text {ix }} \neq \emptyset$. Moreover, $\{\mathrm{Q}$ is (w.P.o).

Theorem 2.7. [20] Let $\mathcal{Q}$ be a multivalued mapping from a complete metric space $(\mathfrak{M}, \delta)$ to $\mathfrak{P}_{c l}(\mathfrak{M})$, and $\lambda \in \mathfrak{S}$. If $\mathcal{Q}$ forms a multivalued $\lambda$-contraction, then

(1) $h$ is a m.w.P. operator;

(2) If additionally $\lambda(\kappa \tau) \leq \kappa \lambda(\tau)$ for every $\tau \in \mathbb{R}^{+}$(where $\kappa>1$ ), then $h$ is a $\varphi$-m.w.P. operator, with $\varphi(\tau):=\tau+\sum_{n=1}^{\infty} \lambda n(\tau)$, for each $\tau \in \mathbb{R}^{+} ;$

(3) Let $S: \mathfrak{M} \rightarrow \mathfrak{P}_{c l}(\mathfrak{M})$ be a $\lambda$-contraction and $\eta>0$ be such that $H_{\delta}(S(\tau), Q(\tau)) \leq \eta$ for each $\tau \in E$. Suppose that $\lambda(\kappa \tau) \leq \kappa \lambda(\tau)$ for every $\tau \in \mathbb{R}^{+}$(where $\kappa>1$ ). Then,

$$
H_{\delta}\left(\mathcal{F}_{i \chi_{S}}, \mathcal{F}_{i \chi_{F}}\right) \leq \varphi(\eta)
$$

For $\varepsilon \in \mathbb{R}$, we set

$$
[\varepsilon]_{q}=\frac{1-q^{\varepsilon}}{1-q} .
$$

Definition 2.8. [15] The q-derivative of order $n \in \mathbb{N}$ of $\mathfrak{g}: \mathfrak{J} \rightarrow \mathbb{R}$ is described as $\left(D_{q}^{0} \mathfrak{g}\right)(\tau)=\mathfrak{g}(\tau)$,

$$
\left(D_{q} \mathfrak{g}\right)(\tau):=\left(D_{q}^{1} \mathfrak{g}\right)(\tau)=\frac{\mathfrak{g}(\tau)-\mathfrak{g}(q \tau)}{(1-q) \tau} ; \tau \neq 0, \quad\left(D_{q} \mathfrak{g}\right)(0)=\lim _{t \rightarrow 0}\left(D_{q} \mathfrak{g}\right)(\tau)
$$

and

$$
\left(D_{q}^{n} \mathfrak{g}\right)(\tau)=\left(D_{q} D_{q}^{n-1} \mathfrak{g}\right)(\tau) ; \tau \in \mathfrak{J}, n \in\{1,2, \ldots\}
$$


Set $I_{s}:=\left\{s q^{n}: n \in \mathbb{N}\right\} \cup\{0\}$.

Definition 2.9. [15] The q-integral of $\mathfrak{g}: I_{s} \rightarrow \mathbb{R}$ is described as

$$
\left(I_{q} \mathfrak{g}\right)(s)=\int_{0}^{s} \mathfrak{g}(\tau) d_{q} \tau=\sum_{n=0}^{\infty} t(1-q) q^{n} \mathfrak{g}\left(s q^{n}\right)
$$

$\left(D_{q} I_{q} \mathfrak{g}\right)(s)=\mathfrak{g}(s)$, while if $\mathfrak{g}$ is continuous at 0 , then

$$
\left(I_{q} D_{q} \mathfrak{g}\right)(s)=\mathfrak{g}(s)-\mathfrak{g}(0) .
$$

Definition 2.10. [8] The Riemann-Liouville fractional q-integral of order $\alpha \in$ $\mathbb{R}_{+}:=[0, \infty)$ of a function $\mathfrak{g}: \mathfrak{J} \rightarrow \mathbb{R}$ is defined by $\left(I_{q}^{0} \mathfrak{g}\right)(s)=\mathfrak{g}(s)$, and

$$
\left(I_{q}^{\alpha} \mathfrak{g}\right)(s)=\int_{0}^{t} \frac{(s-q \tau)^{(\alpha-1)}}{\Gamma_{q}(\alpha)} \mathfrak{g}(\tau) d_{q} \tau ; t \in \mathfrak{J} .
$$

Definition 2.11. [25] The Caputo fractional q-derivative of order $\alpha \in \mathbb{R}_{+}$of a function $\mathfrak{g}: \mathfrak{J} \rightarrow \mathbb{R}$ is defined by $\left({ }^{C} D_{q}^{0} \mathfrak{g}\right)(s)=\mathfrak{g}(s)$, and

$$
\left({ }^{C} D_{q}^{\alpha} \mathfrak{g}\right)(s)=\left(I_{q}^{[\alpha]-\alpha} D_{q}^{[\alpha]} \mathfrak{g}\right)(s) ; s \in \mathfrak{J} .
$$

Lemma 2.12. [25] Let $\alpha \in \mathbb{R}_{+}$. Then

$$
\left(I_{q}^{\alpha}{ }^{C} D_{q}^{\alpha} \mathfrak{g}\right)(s)=\mathfrak{g}(s)-\sum_{k=0}^{[\alpha]-1} \frac{t^{k}}{\Gamma_{q}(1+k)}\left(D_{q}^{k} \mathfrak{g}\right)(0)
$$

In particular, if $\alpha \in(0,1)$, then

$$
\left(I_{q}^{\alpha}{ }^{C} D_{q}^{\alpha} \mathfrak{g}\right)(s)=\mathfrak{g}(s)-\mathfrak{g}(0) .
$$

Lemma 2.13. Assume that $S_{F \circ \mathfrak{g}} \subset C(\mathfrak{J})$ for each $\mathfrak{g} \in C(\mathfrak{J})$. Then (1.1)-(1.2) is equivalent to $\mathfrak{g} \in \mathcal{Q}(\mathfrak{g})$, where $\mathcal{Q}: C(\mathfrak{J}) \rightarrow \mathfrak{P}(C(\mathfrak{J}))$ is the multi-function described as

$$
(\mathcal{Q g})(\tau)=\left\{\mathfrak{g}_{0}+\left(I_{q}^{\alpha} g\right)(\tau): g \in S_{F \circ \mathfrak{g}}\right\}
$$

In this manuscript, we launch the study of the Ulam stability for Caputo fractional $q$-difference inclusions and related coupled systems via Picard operators theory, and it is structured as follows: Section 2 the first main result, existence and stability of (1.1) and (1.2), is expressed. Additionally, in Section 3; we obtain similar results for the coupled system (1.3)-(1.4). Lastly, in Section 4 an example is expressed to indicate the applicability of the derived theorem of the paper. 


\section{Caputo Fractional $q$-Difference Inclusions}

Definition 3.1. $\mathfrak{g}$ is a solution of (1.1)-(1.2) if it achieves the condition (1.2), and the equation $\mathfrak{g}(\tau)=\mathfrak{g}_{0}+\left(I_{q}^{\alpha} g\right)(\tau)$ on $\mathfrak{J}$, where $g \in S_{F \circ \mathfrak{g}}$.

$\left(H_{1}\right)$ The multifunction $\tau \longmapsto F(\tau, \mathfrak{g})$ is jointly measurable for each $\mathfrak{g} \in \mathbb{R}$

$\left(H_{2}\right)$ The multifunction $\mathfrak{g} \longmapsto F(\tau, \mathfrak{g})$ is l.s.c. for a.a. $\tau \in \mathfrak{J}$;

$\left(H_{3}\right)$ There exists a function $\varrho \in L^{\infty}\left(\mathfrak{J}, \mathbb{R}_{+}\right)$and $\lambda \in \mathfrak{S}$ so that

$$
H_{\delta}(F(\tau, \mathfrak{g}), F(\tau, \overline{\mathfrak{g}})) \leq \varrho(\tau) \lambda(|\mathfrak{g}-\overline{\mathfrak{g}}|),
$$

and

$$
\frac{T^{\alpha}\|\varrho\|_{L^{\infty}}}{\Gamma_{q}(1+\alpha)} \leq 1
$$

for almost all $\tau \in \mathfrak{J}$, and each $\mathfrak{g}, \overline{\mathfrak{g}} \in \mathbb{R}$;

$\left(H_{4}\right)$ There exists $q \in L^{1}\left(\mathfrak{J}, \mathbb{R}_{+}\right)$such that

$$
F(\tau, \mathfrak{g}) \subset q(\tau) B_{0},
$$

where $B_{0}=\left\{\mathfrak{g} \in C(\mathfrak{J}):\|\mathfrak{g}\|_{C}<1\right\}$, for almost all $\tau \in \mathfrak{J}$ and each $\mathfrak{g} \in \mathbb{R}$.

A self-mapping $\lambda$ on $[0, \infty)$ is called quasi-homogenous function if If $\lambda(\mathfrak{z} \tau) \leq$ $\mathfrak{d} \lambda(\tau)$ for every $\tau \in \mathbb{R}^{+}$, where $\mathfrak{d}>1$.

Theorem 3.2. Assume $\left(H_{1}\right)-\left(H_{4}\right)$ hold. Then:

(a) Pb. (1.1)-(1.2) admits least one solution and $\mathcal{Q}$ is a m.w.P.o.;

(b) Furthermore, if $\lambda$ is quasi-homogenous, then Pb. (1.1)-(1.2) is g.U.t stable, and $N$ is a $\varphi$-m.w.P.o., with

$$
\varphi(\tau):=t+\sum_{n=1}^{\infty} \lambda^{n}(\tau), \tau \in \mathbb{R}^{+} .
$$

Proof. First, we assert $\mathcal{Q}(\mathfrak{g}) \in \mathfrak{P}_{c p}(C(\mathfrak{J}))$ for each $\mathfrak{g} \in C(\mathfrak{J})$.

For each $\mathfrak{g} \in C(\mathfrak{J})$ there exists $f \in S_{F \circ \mathfrak{g}}$, (see [33]). Thus $\nu(\tau)=\mathfrak{g}_{0}+\left(I_{q}^{\alpha} f\right)(\tau)$ verify $\nu \in \mathcal{Q}(\mathfrak{g})$. From $\left(H_{1}\right)$ and $\left(H_{4}\right)$, via Theorem 8.6.3. in [11], the set $\mathcal{Q}(\mathfrak{g})$ is compact, for each $\mathfrak{g} \in C(\mathfrak{\mathfrak { J }})$.

Next, we assert $H_{\delta}(\mathcal{Q}(\mathfrak{g}), Q(\overline{\mathfrak{g}})) \leq \lambda\left(\|\mathfrak{g}-\overline{\mathfrak{g}}\|_{C}\right)$ for each $\mathfrak{g}, \overline{\mathfrak{g}} \in C(\mathfrak{\mathfrak { J }})$. Let $\mathfrak{g}, \overline{\mathfrak{g}} \in C(\mathfrak{J})$ and $h \in \mathcal{Q}(\mathfrak{g})$. Then, there exists $f \in S_{F \circ \mathfrak{g}}$, with

$$
h(\tau)=\mathfrak{g}_{0}+\left(I_{q}^{\alpha} f\right)(\tau) .
$$


We get, from $\left(H_{3}\right)$

$$
H_{\delta}(F(\tau, \mathfrak{g}(\tau)), F(\tau, \overline{\mathfrak{g}}(\tau))) \leq \varrho(\tau) \lambda\left(\|\mathfrak{g}-\overline{\mathfrak{g}}\|_{C}\right) .
$$

Consequently, there is $w \in S_{F \circ \bar{g}}$, with

$$
|f(\tau)-w(\tau)| \leq \varrho(\tau) \lambda\left(\|\mathfrak{g}-\overline{\mathfrak{g}}\|_{C}\right) ; \tau \in I .
$$

Construct a map $\mathfrak{G}: \mathfrak{J} \rightarrow \mathfrak{P}(\mathbb{R})$ by

$$
\mathfrak{G}(\tau)=\left\{\mathfrak{b} \in \mathbb{R}:|f(\tau)-\mathfrak{b}(\tau)| \leq \varrho(\tau) \lambda\left(\|\mathfrak{g}-\overline{\mathfrak{g}}\|_{C}\right)\right\} .
$$

Due to the measurability of $\mathfrak{g}(\tau)=\mathfrak{G}(\tau) \cap F(\tau, \overline{\mathfrak{g}}(\tau))$ (Proposition III.4 in [13]), then there is $\bar{f}$ which is a measurable selection function for $\mathfrak{g}$. Thus, $\bar{f} \in S_{F \circ \overline{\mathfrak{g}}}$, and for each $\tau \in \mathfrak{J}$,

$$
|f(\tau)-\bar{f}(\tau)| \leq \varrho(\tau) \lambda\left(\| \mathfrak{g}-\left.\overline{\mathfrak{g}}\right|_{C}\right)
$$

Let the function

$$
\bar{h}(\tau)=\mathfrak{g}_{0}+\left(I_{q}^{\alpha} \bar{f}\right)(\tau) .
$$

Then

$$
\begin{aligned}
& |h(\tau)-\bar{h}(\tau)| \leq I_{q}^{\alpha}|f(\tau)-\bar{f}(\tau)| \\
& \leq I_{q}^{\alpha}\left(\varrho(\tau) \lambda\left(\|\mathfrak{g}-\overline{\mathfrak{g}}\|_{C}\right)\right) \\
& \leq\|\varrho\|_{L^{\infty}} \lambda\left(\|\mathfrak{g}-\overline{\mathfrak{g}}\|_{C}\right)\left(\int_{0}^{\tau} \frac{|\tau-q s|^{(\alpha-1)}}{\Gamma_{q}(\alpha)} d_{q} s\right) \\
& \leq \frac{T^{\alpha}\|\varrho\|_{L^{\infty}}}{\Gamma_{q}(1+\alpha)} \lambda\left(\|\mathfrak{g}-\overline{\mathfrak{g}}\|_{C}\right) .
\end{aligned}
$$

Thus, from (4.2) yields

$$
\|h-\bar{h}\|_{C} \leq \lambda\left(\|\mathfrak{g}-\overline{\mathfrak{g}}\|_{C}\right) .
$$

By verbatim with changing the roles of $\mathfrak{g}$ and $\mathfrak{g}$, it yields

$$
H_{\delta}(\mathcal{Q}(\mathfrak{g}), \mathcal{Q}(\overline{\mathfrak{g}})) \leq \lambda\left(\|\mathfrak{g}-\overline{\mathfrak{g}}\|_{C}\right) .
$$

Hence, $Q$ is a $\lambda$-contraction.

(a) Lemma 2.6 infer that $\mathcal{Q}$ possesses a fixed point on $\mathfrak{J}$, and from [Theorem 2.7, (i)] we conclude that 2 is a m.w.P.o.

(b) The problem (1.1)-(1.2) is g.U-H stable. For clarification, for $\varepsilon>0$ and $\nu \in C(\mathfrak{J})$ there is $\mathfrak{g} \in C(\mathfrak{J})$ so that

$$
\mathfrak{g}(\tau)-\mathfrak{g}_{0} \in\left(I_{q}^{\alpha} F\right)(\tau, \nu(\tau)) ; \tau \in \mathfrak{J},
$$


and

$$
\|\mathfrak{g}-\nu\|_{C} \leq \varepsilon
$$

where

$$
\left(I_{q}^{\alpha} F\right)(\tau, \nu(\tau))=\left\{\left(I_{q}^{\alpha} w\right)(\tau) ; w \in S_{F \circ \nu}\right\} ; \tau \in \mathfrak{J} .
$$

Then $H_{\delta}(y, \mathcal{Q}(y)) \leq \varepsilon$. Moreover, the multivalued map $\mathcal{Q}$ is $\lambda$-contraction, and from [Theorem 2.7, (i)-(ii)], $\mathcal{Q}$ is a $\varphi$-m.w.P.o. Tus, $\mathfrak{g} \in \mathcal{Q}(\mathfrak{g})$ is g.U-H stable. Hence, our problem (1.1)-(1.2) is g.U-H stable. Theorem 2.7,(iii) concludes the result.

\section{Caputo Fractional $q$-Difference Inclusions}

Ulam stability of the problem (1.1)-(1.2) shall be discussed in this section.

Definition 4.1. If a continuous $\mathfrak{g}$ along the initial condition (1.2) achieve $\mathfrak{g}(\mathfrak{t})=\mathfrak{g}_{0}+\left(\mathbb{I}_{q}^{\alpha} g\right)(\mathfrak{t})$ on $\mathfrak{J}$, where $g \in S_{F \circ \mathfrak{g}}$, then we say that it is a solution of the problem (1.1)-(1.2)

Now, we present requirements for both Ulam stability of problem (1.1)$(1.2)$.

The following are the basic requirements for our aim:

$\left(H_{1}\right)$ The multifunction $\mathfrak{t} \longmapsto F(\mathfrak{t}, \mathfrak{g})$ is jointly measurable for each $\mathfrak{g} \in \mathbb{R}$

$\left(H_{2}\right)$ The multifunction $\mathfrak{g} \longmapsto F(\mathfrak{t}, \mathfrak{g})$ is lower semi-continuous for almost all $\mathfrak{t} \in \mathfrak{J}$

$\left(H_{3}\right)$ There exists $\rho \in L^{\infty}\left(\mathfrak{J}, \mathbb{R}_{+}\right)$and $\varphi \in \mathfrak{S}$ so that for for almost all $\mathfrak{t} \in \mathfrak{J}$, and each $\mathfrak{g}, \overline{\mathfrak{g}} \in \mathbb{R}$, we have

$$
H_{d}(F(\mathfrak{t}, \mathfrak{g}), F(\mathfrak{t}, \overline{\mathfrak{g}})) \leq \varrho(\mathfrak{t}) \varphi(|\mathfrak{g}-\overline{\mathfrak{g}}|),
$$

and

$$
\frac{T^{\alpha}\|\varrho\|_{L^{\infty}}}{\Gamma_{q}(1+\alpha)} \leq 1
$$

$\left(H_{4}\right)$ we have $F(\mathfrak{t}, \mathfrak{g}) \subset q(\mathfrak{t}) B(0,1)$, for almost all $\mathfrak{t} \in \mathfrak{J}$ and each $\mathfrak{g} \in \mathbb{R}$, where $q: \mathfrak{J} \rightarrow \mathbb{R}$ is integrable and $B(0,1)=\left\{\mathfrak{g} \in C(\mathfrak{J}):\|\mathfrak{g}\|_{C}<1\right\}$

Theorem 4.2. Suppose that $\left(H_{1}\right)-\left(H_{4}\right)$ are achieved by the multifunction $F: \mathfrak{J} \times \mathbb{R} \rightarrow \mathfrak{P}_{c p}(\mathbb{R})$ Then,

(a) Problem (1.1)-(1.2) possesses a solution and $\mathcal{Q}$ is a m.w.P.o; 
(b) In addition, if $\lambda$ is quasi-homogenous, then the problem defined by (1.1)(1.2) is g.U.t. stable, and $\mathfrak{Q}$ is a $\lambda$-m.w.P.o, where $\lambda$ is described as $\lambda(\mathfrak{t}):=t+\sum_{n=1}^{\infty} \varphi^{n}(\mathfrak{t})$, for each $\mathfrak{t} \in[0, \infty)$.

Remark 4.3. Note that $S_{F \circ \mathfrak{g}}$ is nonempty for all $\mathfrak{g} \in C(\mathfrak{J})$ on account of $\left(H_{1}\right) F$ has a measurable selection (see [13], Theorem III.6)

Proof. Let $\mathcal{Q}$ a mapping as described in Lemma 2.13. We assert that it achieves the hypothesis of Theorem 2.7. We assert first that $\mathcal{Q}(x) \in \mathfrak{P}_{c p}(C(\mathfrak{J}))$ for each $\mathfrak{g} \in C(\mathfrak{J})$.

On account of Theorem 2 in [33], for each $\mathfrak{g} \in C(\mathfrak{J})$ there is $f \in S_{F \circ \mathfrak{g}}$, for all $\mathfrak{t} \in \mathfrak{J}$. Then, $\nu(\mathfrak{t})=\mathfrak{g}_{0}+\left(\mathbb{I}_{q}^{\alpha} f\right)(\mathfrak{t})$ has the property $\nu \in \mathcal{Q}(\mathfrak{g})$. In addition, taking $\left(H_{1}\right)$ and $\left(H_{4}\right)$, together with Theorem 8.6.3. in [11], we find that for each $\mathfrak{g} \in C(\mathfrak{J})$, the set $\mathcal{Q}(\mathfrak{g})$ is compact.

Next, we assert that $H_{d}(\mathcal{Q}(\mathfrak{g}), \mathcal{Q}(\overline{\mathfrak{g}})) \leq \varphi\left(\|\mathfrak{g}-\overline{\mathfrak{g}}\|_{C}\right)$ for each $\mathfrak{g}, \overline{\mathfrak{g}} \in C(\mathfrak{J})$. Let $\mathfrak{g}, \overline{\mathfrak{g}} \in C(\mathfrak{J})$ and $h \in \mathcal{Q}(\mathfrak{g})$. So, there is $f \in S_{F \circ \mathfrak{g}}$, so that

$$
h(\mathfrak{t})=\mathfrak{g}_{0}+\left(\mathbb{I}_{q}^{\alpha} f\right)(\mathfrak{t}),
$$

for each $\mathfrak{t} \in \mathfrak{J}$. Due to $\left(H_{3}\right)$, we have

$$
H_{d}(F(\mathfrak{t}, \mathfrak{g}(\mathfrak{t})), F(\mathfrak{t}, \overline{\mathfrak{g}}(\mathfrak{t}))) \leq \varrho(\mathfrak{t}) \varphi\left(\|\mathfrak{g}-\overline{\mathfrak{g}}\|_{C}\right)
$$

Consequently, there is $w \in S_{F \circ \overline{\mathfrak{g}}}$, with

$$
|f(\mathfrak{t})-w(\mathfrak{t})| \leq \varrho(\mathfrak{t}) \varphi\left(\|\mathfrak{g}-\overline{\mathfrak{g}}\|_{C}\right) ; \mathfrak{t} \in \mathfrak{J} .
$$

We set $\mathfrak{G}: \mathfrak{J} \rightarrow \mathfrak{P}(\mathbb{R})$ as follows

$$
\mathfrak{G}(\mathfrak{t})=\left\{w \in \mathbb{R}:|f(\mathfrak{t})-w(\mathfrak{t})| \leq \varrho(\mathfrak{t}) \varphi\left(\|\mathfrak{g}-\overline{\mathfrak{g}}\|_{C}\right)\right\}
$$

Note that $\mathfrak{g}(\mathfrak{t})=\mathfrak{G}(\mathfrak{t}) \cap F(\mathfrak{t}, \overline{\mathfrak{g}}(\mathfrak{t}))$ is a measurable multivalued operator due to Proposition III.4 in [13]. Consequently, there is a measurable selection function $\bar{f}$ for $\mathfrak{g}$. Thus, $\bar{f} \in S_{F \circ \overline{\mathfrak{g}}}$, and

$$
|f(\mathfrak{t})-\bar{f}(\mathfrak{t})| \leq \varrho(\mathfrak{t}) \varphi\left(\| \mathfrak{g}-\left.\overline{\mathfrak{g}}\right|_{C}\right),
$$

for all $\mathfrak{t} \in \mathfrak{J}$. Define

$$
\bar{h}(\mathfrak{t})=\mathfrak{g}_{0}+\left(\mathbb{I}_{q}^{\alpha} \bar{f}\right)(\mathfrak{t}),
$$


for each $\mathfrak{t} \in \mathfrak{J}$. Consequently, we find

$$
\begin{aligned}
& |h(\mathfrak{t})-\bar{h}(\mathfrak{t})| \leq \mathfrak{J}_{q}^{\alpha}|f(\mathfrak{t})-\bar{f}(\mathfrak{t})| \\
& \leq \mathfrak{J}_{q}^{\alpha}\left(\varrho(\mathfrak{t}) \varphi\left(\|\mathfrak{g}-\overline{\mathfrak{g}}\|_{C}\right)\right) \\
& \leq\|\varrho\|_{L^{\infty}} \varphi\left(\|\mathfrak{g}-\overline{\mathfrak{g}}\|_{C}\right)\left(\int_{0}^{\mathfrak{t}} \frac{|\mathfrak{t}-q s|^{(\alpha-1)}}{\Gamma_{q}(\alpha)} d_{q} s\right) \\
& \leq \frac{T^{\alpha}\|\varrho\|_{L^{\infty}}}{\Gamma_{q}(1+\alpha)} \varphi\left(\|\mathfrak{g}-\overline{\mathfrak{g}}\|_{C}\right),
\end{aligned}
$$

for each $t \in \mathfrak{J}$. On account of (4.2), we find

$$
\|h-\bar{h}\|_{C} \leq \varphi\left(\|\mathfrak{g}-\overline{\mathfrak{g}}\|_{C}\right) .
$$

Regarding the analogy, changing the roles of $\mathfrak{g}$ and $\overline{\mathfrak{g}}$, yields

$$
H_{d}(\mathcal{Q}(\mathfrak{g}), \mathcal{Q}(\overline{\mathfrak{g}})) \leq \varphi\left(\|\mathfrak{g}-\overline{\mathfrak{g}}\|_{C}\right) .
$$

As a result, $\mathcal{Q}$ is a $\varphi$-contraction.

By taking Lemma 2.6 into account, we deduce that fixed point of $Q$ possesses a solution of the inclusion (1.1)-(1.2) on $\mathfrak{J}$. Further, [Theorem 2.7, (i)] yields that $\mathcal{Q}$ is a m.w.P.o.

Now, we assert that the problem (1.1)-(1.2) is g.U.t stable.

For this purpose, take $\epsilon>0$ and $\nu \in C(\mathfrak{J})$ for which there is $\mathfrak{g} \in C(\mathfrak{J})$ so that

$$
\mathfrak{g}(\mathfrak{t})-\mathfrak{g}_{0} \in\left(\mathfrak{J}_{q}^{\alpha} F\right)(\mathfrak{t}, \vartheta(\mathfrak{t})) ; \mathfrak{t} \in \mathfrak{J},
$$

and

with

$$
\|\mathfrak{g}-\nu\|_{C} \leq \epsilon
$$

$$
\left(\mathfrak{J}_{q}^{\alpha} F\right)(\mathfrak{t}, \nu(\mathfrak{t}))=\left\{\left(\mathfrak{J}_{q}^{\alpha} w\right)(\mathfrak{t}) ; w \in S_{F \circ \nu}\right\} ; \mathfrak{t} \in \mathfrak{J} .
$$

Then $H_{d}(y, \mathcal{Q}(y)) \leq \epsilon$. In addition, we conclude that $\mathcal{Q}$ is a multivalued $\varphi$-contraction. Regarding [Theorem 2.7, (i)-(ii)], we deduce that $\mathcal{Q}$ is a $\Psi$ m.w.P.o. Thus, the fixed point problem $\mathfrak{g} \in \mathcal{Q}(\mathfrak{g})$ is g.U.t. stable. In conclusion, the problem (1.1)-(1.2) is g.U.t. stable. The rest follows from [Theorem $2.7,($ iii) $]$.

\section{Coupled System of Caputo Fractional q-Difference Inclusions}

This section is devoted for the existence, uniqueness and Ulam stability of (1.3)-(1.4). Here, $\mathcal{C}:=C(\mathfrak{J}) \times C(\mathfrak{J})$ denotes the Banach space with the norm

$$
\|(\mathfrak{g}, \nu)\|_{\mathfrak{e}}=\|\mathfrak{g}\|_{C}+\|\nu\|_{C} .
$$


Lemma 5.1. Let $G: \mathcal{C} \rightarrow \mathcal{C}$ described as

$$
\left.\left(G\left(\mathfrak{g}_{1}, \mathfrak{g}_{2}\right)\right)(\mathfrak{t})=\left(\left(G_{1} \mathfrak{g}_{1}\right)(\mathfrak{t}), G_{2} \mathfrak{g}-2\right)(\mathfrak{t})\right) ; \mathfrak{t} \in \mathfrak{J},
$$

where $G_{i}: C(\mathfrak{J}) \rightarrow C(\mathfrak{J}) ; i=1,2$, are defined by

$$
\left(G_{i}\left(\mathfrak{g}_{1}, \mathfrak{g}_{2}\right)\right)(\mathfrak{t})=\mathfrak{i}_{i}+\left(\mathfrak{J}_{q_{i}}^{\alpha_{i}} g_{i}\right)(\mathfrak{t}) ; \mathfrak{t} \in \mathfrak{J}
$$

where $g_{i} \in S_{F_{i} \circ \mathfrak{g}_{i}} ; i=1,2$. Then, fixed points of $G$ form the solutions of the system (1.3)-(1.4).

Definition 5.2. By a coupled solutions of problem (1.3)-(1.4) we mean a continuous coupled functions $\left(\mathfrak{g}_{1}, \mathfrak{g}_{2}\right)$ those satisfy the initial condition (1.4), and the equations $\mathfrak{g}_{i}(\mathfrak{t})=\mathfrak{i}_{i}+\left(\mathfrak{J}_{q_{i}}^{\alpha_{i}} \nu_{i}\right)(\mathfrak{t})$ on $\mathfrak{J}$, where $\nu_{i} \in S_{F_{i} \circ \mathfrak{g}_{i}} ; i=1,2$.

Keeping Lemma 5.1 on mind, we shall investigate the existence and Ulam stability of (1.3)-(1.4), as in Theorem 4.2.

Theorem 5.3. Assume that the multifunctions $F_{i}: \mathfrak{J} \times \mathbb{R} \times \mathbb{R} \rightarrow \mathcal{N}_{c p}(\mathbb{R})$ satisfy the following hypotheses

$\left(H_{01}\right)$ The multifunctions $\mathfrak{t} \longmapsto F_{i}\left(\mathfrak{t}, \mathfrak{g}_{1}, \mathfrak{g}_{2}\right)$ are jointly measurable for each $\mathfrak{g}_{i} \in \mathbb{R} ; i=1,2$,

$\left(H_{02}\right)$ The multifunctions $\mathfrak{g}_{i} \longmapsto F\left(\mathfrak{t}, \mathfrak{g}_{1}, \mathfrak{g}_{2}\right)$ are lower semi-continuous for almost all $\mathfrak{t} \in \mathfrak{J}$;

$\left(H_{03}\right)$ There exist $p_{i} \in L^{\infty}\left(\mathfrak{J}, \mathbb{R}_{+}\right)$and $\varphi_{i}: \in \mathfrak{S}$ such that

$$
H_{d}\left(F_{i}\left(\mathfrak{t}, \mathfrak{g}_{1}, \mathfrak{g}_{2}\right), F_{i}\left(\mathfrak{t}, \overline{\mathfrak{g}_{1}}, \overline{\mathfrak{g}_{2}}\right) \leq p_{i}(\mathfrak{t}) \varphi_{i}\left(\left|\mathfrak{g}_{i}-\overline{\mathfrak{g}_{i}}\right|\right),\right.
$$

and

$$
\frac{T^{\alpha_{i}}\left\|p_{i}\right\|_{L^{\infty}}}{\Gamma_{q}\left(1+\alpha_{i}\right)} \leq 1
$$

or for almost all $\mathfrak{t} \in \mathfrak{J}$, and each $\mathfrak{g}_{1}, \mathfrak{g}_{2}, \overline{\mathfrak{g}_{1}}, \overline{\mathfrak{g}_{2}} \in \mathbb{R}$.

$\left(H_{04}\right)$ There exist integrable functions $q_{i}: \mathfrak{J} \rightarrow \mathbb{R}$ such that for almost all $\mathfrak{t} \in \mathfrak{J}$ and each $\mathfrak{g}_{i} \in \mathbb{R} ; i=1,2$, we have

$$
F_{i}\left(\mathfrak{t}, \mathfrak{g}_{1}, \mathfrak{g}_{2}\right) \subset q_{i}(\mathfrak{t}) B(0,1),
$$

where $B(0,1)=\left\{\nu \in C(\mathfrak{J}):\|\nu\|_{C}<1\right\}$.

Then, we have

(a) Problem (1.3)-(1.4) possess a solution and $G$ is a m.w.P.o; 
(b) In addition, if each $\varphi_{i}$ is quasi-homogenous, $(i=1,2$,$) then the problem$ (1.3)-(1.4) is g. U. t. stable, and $G$ is a $\Psi$-m.w.P.o, with $\Psi=\left(\Psi_{1}, \Psi_{2}\right)$ and the the functions $\Psi_{i} ; i=1,2$ defined by $\Psi_{i}(\mathfrak{t}):=\mathfrak{t}+\sum_{n=1}^{\infty} \varphi_{i}^{n}(\mathfrak{t})$, for each $\mathfrak{t} \in[0, \infty)$.

\section{An Example}

We aim to deal with the Cauchy problem of Caputo fractional $\frac{1}{4}$-difference inclusion

$$
\left\{\begin{array}{l}
\left({ }^{C} \mathfrak{D} \frac{1}{4}^{\frac{1}{2}} \mathfrak{g}\right)(\mathfrak{t}) \in F(\mathfrak{t}, \mathfrak{g}(\mathfrak{t})) ; \mathfrak{t} \in[0,1], \\
\left.\mathfrak{g}(\mathfrak{t})\right|_{\mathfrak{t}=0}=1
\end{array}\right.
$$

for

$$
F(\mathfrak{t}, \mathfrak{g}(\mathfrak{t}))=\left\{\nu \in C([0,1], \mathbb{R}):\left|f_{1}(\mathfrak{t}, \mathfrak{g}(\mathfrak{t}))\right| \leq|\nu| \leq\left|f_{2}(\mathfrak{t}, \mathfrak{g}(\mathfrak{t}))\right|\right\} ; \mathfrak{t} \in[0,1],
$$

where $f_{1}, f_{2}:[0,1] \times \mathbb{R} \rightarrow \mathbb{R}$, such that

$$
f_{1}(\mathfrak{t}, \mathfrak{g}(\mathfrak{t}))=\frac{\mathfrak{t}^{2} \mathfrak{g}(\mathfrak{t})}{(1+|\mathfrak{g}(\mathfrak{t})|) e^{10+\mathfrak{t}}}, \quad f_{2}(\mathfrak{t}, \mathfrak{g}(\mathfrak{t}))=\frac{\mathfrak{t}^{2} \mathfrak{g}(\mathfrak{t})}{e^{10+\mathfrak{t}}}
$$

Set $\alpha=\frac{1}{2}$ and suppose that $F$ is both convex and closed multivalued function. Notice that the solutions of the problem (6.1) are the solutions

$$
\mathfrak{g} \in A(\mathfrak{g}) \text { ( the fixed point inclusion) }
$$

where the multifunction operator $A: C([0,1], \mathbb{R}) \rightarrow \mathfrak{P}(C([0,1], \mathbb{R}))$ is described as

$$
(A \mathfrak{g})(\mathfrak{t})=\left\{1+\left(\mathfrak{J}_{\frac{1}{4}}^{\frac{1}{2}} f\right)(\mathfrak{t}) ; f \in S_{F \circ \mathfrak{g}}\right\} ; \mathfrak{t} \in[0,1] .
$$

For each $\mathfrak{t} \in[0,1]$ and all $z_{1}, z_{2} \in C([0,1], \mathbb{R})$, we have

$$
\left\|f_{2}\left(\mathfrak{t}, z_{2}\right)-f_{1}\left(\mathfrak{t}, z_{1}\right)\right\|_{C} \leq \mathfrak{t}^{2} e^{-10-\mathfrak{t}}\left\|z_{2}-z_{1}\right\|_{C} .
$$

Consequently, we conclude that all hypotheses $\left(H_{1}\right)-\left(H_{3}\right)$ are achieved with $\varrho(\mathfrak{t})=\mathfrak{t}^{2} e^{-10-\mathfrak{t}}$.

As a next step, we indicate that condition (4.2) is fulfilled for $T=1$. For clarification, we note that $\|\varrho\|_{L^{\infty}}=e^{-9}, \Gamma_{\frac{1}{4}}\left(1+\frac{1}{2}\right)>\frac{1}{2}$. After an elementary calculation, one can get that

$$
\Delta:=\frac{T^{\frac{3}{4}}\|\varrho\|_{L^{\infty}}}{\Gamma_{\frac{1}{4}}\left(1+\frac{1}{2}\right)}<2 e^{-9}<1 .
$$


Furthermore, $\left(H_{4}\right)$ is fulfilled with $q(\mathfrak{t})=\frac{\mathfrak{t}^{2} e^{-10-\mathfrak{t}}}{\|F\|_{\mathfrak{P}}} ; \mathfrak{t} \in[0,1]$, where

$$
\|F\|_{\mathfrak{P}}=\sup \left\{\|f\|_{C}: f \in S_{F \circ \mathfrak{g}}\right\} ; \text { for all } \mathfrak{g} \in C([0,1], \mathbb{R}) .
$$

As a result, Theorem 4.2 implies that:

(a) The problem (6.1) possesses a solution and $A$ is a m.w.P.o.

(b) The function $\varphi(\mathfrak{t})=\Delta \mathfrak{t}$ forms quasi-homogenous. Hence, the problem (6.1) is g.U.t. stable, and $A$ is a $\Psi$-m.w.P.o, with the function $\Psi$ defined by $\Psi(\mathfrak{t}):=t+(1-\Delta \mathfrak{t})^{-1}$, for each $\mathfrak{t} \in\left[0, \Delta^{-1}\right)$.

\section{References}

[1] S. Abbas, W.A. Albarakati, M. Benchohra and A. Petruşel, Existence and Ulam stability results for Hadamard partial fractional integral inclusions via Picard operators, Stud. Univ. Babeş-Bolyai Math., 61 (4) (2016), 409420.

[2] S. Abbas, M. Benchohra and A. Petruşel, Ulam stability for Hilfer type fractional differential inclusions via the weakly Picard operator theory, Frac. Calc. Appl. Anal. 20 (2) (2017), 384-398.

[3] S. Abbas, M. Benchohra and A. Petrussel, Ulam stabilities for partial fractional differential inclusions via Picard Operators, Electron. J. Qual. Theory Differ. Equ., 51 (2014), 1-13.

[4] R. S. Adiguzel, U. Aksoy, E. Karapinar, I.M. Erhan, On the solution of a boundary value problem associated with a fractional differential equation Mathematical Methods in the Applied Sciences, https://doi.org/10.1002/mma.6652

[5] R. S. Adiguzel, U. Aksoy, E. Karapinar, I.M. Erhan, On The Solutions of Fractional Differential Equations via Geraghty Type Hybrid Contractions, Appl. Comput. Math., V.20, N.2, 2021,

[6] H.Afshari, S. Kalantari, E. Karapinar, Solution of fractional differential equations via coupled fixed point, Electronic Journal of Differential Equations, Vol. 2015 (2015), No. 286, pp. 1-12

[7] H. Afshari, E, Karapınar, A discussion on the existence of positive solutions of the boundary value problems via $\phi$-Hilfer fractional derivative on b-metric spaces Advances in Difference Equations volume 2020, Article number: 616 (2020)

[8] R. Agarwal, Certain fractional q-integrals and q-derivatives, Proc. Camb. Philos. Soc. 66 (1969), 365-370. 
[9] A.Abdeljawad, R.P. Agarwal, E. Karapinar, P.S.Kumari, Solutions of he Nonlinear Integral Equation and Fractional Differential Equation Using the Technique of a Fixed Point with a Numerical Experiment in Extended b-Metric Space. Symmetry 2019, 11, 686.

[10] A. Ardjouni, Asymptotic stability in Caputo-Hadamard fractional dynamic equations, Results in Nonlinear Analysis, Volume 4, Issue 2, 2021, $77-86$

[11] J.-P. Aubin, H. Frankowska, Set-Valued Analysis, Birkhauser, Basel, 1990.

[12] R. D. Carmichael, The general theory of linear q-difference equations, American J. Math. 34 (1912), 147-168.

[13] C. Castaing and M. Valadier, Convex Analysis and Measurable Multifunctions, Lecture Notes in Mathematics 580, Springer-Verlag, BerlinHeidelberg-New York, 1977.

[14] K. Deimling, Multivalued Differential Equations, Walter De Gruyter, Berlin-New York, 1992.

[15] V. Kac and P. Cheung, Quantum Calculus. Springer, New York, 2002.

[16] E.Karapinar and A.Fulga, An admissible Hybrid contraction with an Ulam type stability, Demonstr. Math. (2019) ; 52:428-436

[17] E. Karapinar, T.Abdeljawad; F. Jarad, Applying new fixed point theorems on fractional and ordinary differential equations, Advances in Difference Equations, 2019, 2019:421

[18] A.A. Kilbas, H. M. Srivastava, and Juan J. Trujillo, Theory and Applications of Fractional Differential Equations. North-Holland Mathematics Studies, 204. Elsevier Science B.V., Amsterdam, 2006.

[19] M. Kisielewicz, Differential Inclusions and Optimal Control, Kluwer, Dordrecht, The Netherlands, 1991.

[20] V.L. Lazăr, Fixed point theory for multivalued $\varphi$-contractions, Fixed Point Theory Appl. 2011, 2011:50, 1-12.

[21] M. Marin, O. Florea, and S. R.Mahmoud, On temporal behaviour of solutions in thermoelasticity of porous micropolar bodies, An. St. Univ. Ovidius Constanta 22 (1) (2014), 169-188.

[22] K. Sharma and M. Marin, Reflection and transmission of waves from imperfect boundary between two heat conducting micropolar thermoelastic solids, An. St. Univ. Ovidius Constanta, 22(2), 151-175 (2014)

Dissertationes Math. 127 (1975), 1-68.

Pacific J. Math., 30 (1969), 475-488. 
[23] A. Petruşel, Multivalued weakly Picard operators and applications, Sci. Math. Japon. 59 (2004), 167-202.

[24] P.M. Rajkovic, S.D. Marinkovic and M.S. Stankovic, Fractional integrals and derivatives in q-calculus, Appl. Anal. Discrete Math., 1 (2007), 311323.

[25] P.M. Rajkovic, S.D. Marinkovic and M.S. Stankovic, On q-analogues of Caputo derivative and Mittag-Leffler function, Fract. Calc. Appl. Anal., 10 (2007), 359-373.

[26] I. A. Rus, Ulam stability of ordinary differential equations, Studia Univ. Babes-Bolyai, Math. LIV (4)(2009), 125-133.

[27] I. A. Rus, Remarks on Ulam stability of the operatorial equations, Fixed Point Theory 10 (2009), 305-320.

[28] I. A. Rus, Picard operators and applications Sci. Math. Jpn. 58 (2003), $1,191-219$.

[29] I. A. Rus, Some problems in the fixed point theory, Advances in the Theory of Nonlinear Analysis and its Application, Volume 2, Issue 1, 2018, 1 - 10 .

[30] I. A. Rus, Weakly Picard operators and applications, Semin. Fixed Point Theory Cluj-Napoca 2 (2001), 41-58.

[31] I. A. Rus, A. Petrusel, G. Petrusel, Fixed Point Theory, Cluj University Press, Cluj, 2008.

[32] I.A. Rus, A. Petruşel, A. Sîtămărian, Data dependence of the fixed points set of some multivalued weakly Picard operators, Nonlinear Anal. 52 (2003), 1947-1959.

[33] L. Rybinski, On Carathédory type selections, Fund. Math. 125 (1985), 187-193.

[34] I.A. Rus, Some variants of contraction principle in the case of operators with Volterra property: step by step contraction principle, Advances in the Theory of Nonlinear Analysis and its Application, Volume 3, Issue 3, 2019, $111-120$

[35] S. G. Samko, A. A. Kilbas and O. I. Marichev, Fractional Integrals and Derivatives. Theory and Applications, Gordon and Breach, Amsterdam, 1987, Engl. Trans. from the Russian.

[36] G. V. Smirnov, Introduction to the Theory of Differential Inclusions. Graduate Studies in Mathematics, 41. American Mathematical Society, Providence, RI, 2002. 
[37] K. Sharma and M. Marin, Reflection and transmission of waves from imperfect boundary between two heat conducting micropolar thermoelastic solids, An. St. Univ. Ovidius Constanta, 22 (2) (2014), 151-175.

[38] V. E. Tarasov, Fractional Dynamics: Application of Fractional Calculus to Dynamics of Particles, Fields and Media, Springer, Heidelberg; Higher Education Press, Beijing, 2010.

Measures of Noncompactness in Metric Fixed Point Theory, Birkhauser, Basel, 1997.

[39] R. Wȩgrzyk, Fixed point theorems for multifunctions and their applications to functional equations, Dissertationes Math. (Rozprawy Mat.) 201 (1982), $28 \mathrm{pp}$.

[40] Y. Zhou, J.-R. Wang, L. Zhang, Basic Theory of Fractional Differential Equations, Second edition. World Scientific Publishing Co. Pte. Ltd., Hackensack, NJ, 2017.

Saïd ABBAS,

Department of Mathematics,

University of Saïda-Dr. Moulay Tahar,

P.O. Box 138, EN-Nasr, 20000 Saïda, Algeria

Email: abbasmsaid@yahoo.fr, said.abbas@univ-saida.dz

Mouffak BENCHOHRA,

Laboratory of Mathematics,

Djillali Liabes University of Sidi Bel-Abbès,

P.O. Box 89, Sidi Bel-Abbès 22000, Algeria Email: benchohra@yahoo.com

Erdal KARAPINAR,

(1) Division of Applied Mathematics, Thu Dau Mot University, Binh Duong

Province, Vietnam

Email:erdalkarapinar@tdmu.edu.vn

(2) Department of Mathematics, Çankaya University, 06790, Etimesgut, Ankara, Turkey. Email:erdalkarapinar@yahoo.com

(3) Department of Medical Research, China Medical Univer-

sity Hospital, China Medical University, 40402, Taichung, Taiwan

Email:karapinar@mail.cmuh.org.tw 